\title{
Retinal Image Analysis Oriented to the Clinical Task
}

\author{
Andrés G. Marrugo \\ Facultad de Ingeniería, Universidad Tecnológica de Bolívar, Km. 1 vía Turbaco, Cartagena, Colombia \\ Advisor/s: María S. Millán \\ Date and location of PhD thesis defense: 29 November 2013, Universitat Politècnica de Catalunya
}

Received 27th January 2014; accepted 28th May 2014

\section{Introduction}

Ophthalmology can profit greatly from the analysis of digital images because they can aid in establishing an early diagnosis even before the first symptoms appear. This dissertation [1] contributes to the digital analysis of such images and the problems that arise along the imaging pipeline of fundus photography, a field that is commonly referred to as retinal image analysis. We have dealt with and proposed solutions to problems that arise in retinal image acquisition and longitudinal monitoring of retinal disease evolution. Specifically, non-uniform illumination compensation [2], poor image quality [3], automated focusing [4], image segmentation [5], change detection [6], space-invariant (SI) [6] and space-variant (SV) [7] blind deconvolution (BD). Digital retinal image analysis can be effective and cost-efficient for disease management, computer-aided diagnosis, screening and telemedicine and applicable to a variety of disorders such as glaucoma, macular degeneration, and retinopathy [8, 9].

\section{Retinal Image Acquisition}

Acquiring good quality retinal images is important in order to extract meaningful information that is medically relevant. Identifying good quality images is mainly a no-reference quality assessment problem in which there is no ground-truth. In [3] we studied several no-reference quality metrics and found anisotropy to be an important feature to quantify image degradation, specially blurring. The metric based on anisotropy showed the best agreement when compared to the image quality assessment produced by two expert readers.

Along the same line, we proposed a focus measure for non-mydriatic fundus imaging based on the anisotropic properties of image degradation [4]. The proposed focus measure is based on a weighted directional variance of the normalized discrete cosine transform. The normalization is carried out in order to achieve illumination invariance. The focus measure performance was compared, by means of simulated and real images, to other standard focus measures. The results obtained from the analysis of infrared image sequence extracted from the retinal camera showed that the proposed measure outperformed the considered measures in robustness and accuracy. The code is available in [10].

\footnotetext{
Correspondence to: agmarrugo@utbvirtual.edu.co

Recommended for acceptance by Alicia Fornés and Volkmar Frinken ELCVIA ISSN:1577-5097

Published by Computer Vision Center / Universitat Autònoma de Barcelona, Barcelona, Spain
} 

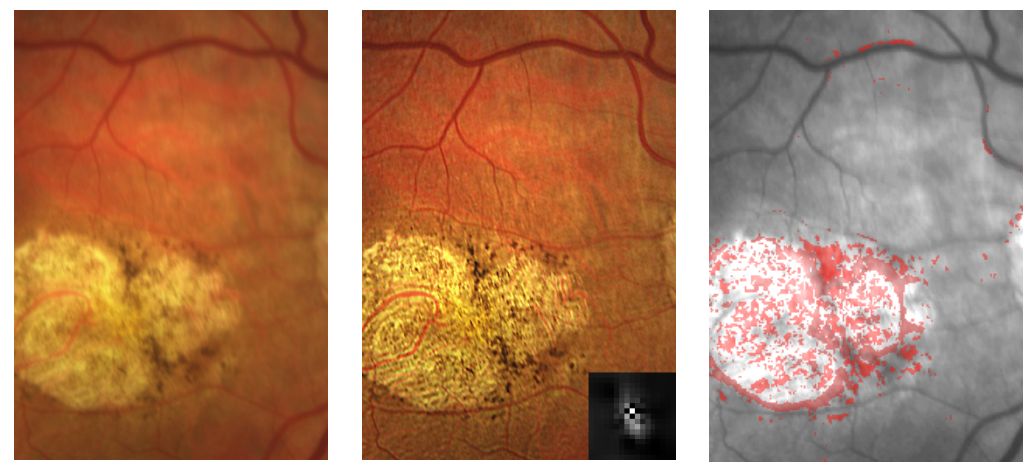

Figure 1. From left to right: degraded retinal image, restored image with estimated PSF, change detection.

\section{Retinal Image Enhancement}

Blur is one of the main image quality degradations in eye fundus imaging. We have addressed this problem by means of $\mathrm{BD}$, in which the blur can be described by convolution with an unknown point-spread function (PSF). In a first approach [6] we considered the blur to be SI and proposed a multichannel BD strategy for the restoration of blurred retinal images. As an unprecedented take on the multichannel BD problem, we use pair of retinal images from the same patient acquired even months apart. We adjust the images (registration, illumination compensation, and structural change detection) [2] so they comply with the proposed degradation model. We developed a strategy for the detection of structural changes between acquisitions (Figure 1), e.g., macular degeneration, hemorrhages, aneurysms, etc. Such changes are masked in the PSF estimation stage. The proposed approach was tested on both synthetically and real degraded images showing a remarkable enhancement evidenced by the increase in visibility of details such as small blood vessels (Figure 1).

The SI approach may fail because the blur changes across the field of view. For that reason, we proposed a SV BD approach [7]. Because the blur changes smoothly we assume the SV PSF to be locally SI. Thus, we can estimate local PSFs by dividing the image into a grid of small patches. We detect possible PSF estimation errors that may arise in regions with scarce structural information. [11] Unlike other works, we do not deconvolve the image per patch and stitch the patches together to produce the restored image. The estimated local PSFs are sewn through interpolation to build the SV PSF. The method was tested on artificially and naturally degraded retinal images obtaining an important enhancement in all cases which can leverage the images' clinical use.

\section{References}

[1] A. G. Marrugo, Ph.D. Thesis "http://hdl . handle.net/10803/134698",

[2] A. G. Marrugo and M. S. Millán, "Retinal image analysis: preprocessing and feature extraction," Journal of Physics: Conference Series, vol. 274, no. 1, p. 012039, 2011.

[3] A. G. Marrugo, M. S. Millán, G. Cristóbal, S. Gabarda, and H. C. Abril, "No-reference Quality Metrics for Eye Fundus Imaging," CAIP 2011, LNCS, vol. 6854, pp. 486-493, 2011.

[4] A. G. Marrugo, M. S. Millán, G. Cristóbal, S. Gabarda, and H. C. Abril, "Anisotropy-based robust focus measure for nonmydriatic retinal imaging," Journal of Biomedical Optics, vol. 17, no. 7, p. 076021, 2012.

[5] A. G. Marrugo and M. S. Millán, "Optic disc segmentation in retinal images," Opt. Pura Apl., vol. 43, pp. 79-86, May 2010.

[6] A. G. Marrugo, M. Sorel, F. Sroubek, and M. S. Millán, "Retinal image restoration by means of blind deconvolution," Journal of Biomedical Optics, vol. 16, no. 11, p. 116016, 2011.

[7] A. G. Marrugo, M. S. Millan, M. Sorel, and F. Sroubek, "Restoration of retinal images with space-variant blur," Journal of Biomedical Optics, vol. 19, no. 1, p. 016023, 2014.

[8] A. G. Marrugo, et al., "Toward computer-assisted diagnosis and telemedicine in ophthalmology," SPIE Newsroom, no. doi: 10.1117/2.1201205.004256, 2012.

[9] A. G. Marrugo, et al., "Image analysis in modern ophthalmology: from acquisition to computer assisted diagnosis and telemedicine," in SPIE Proceedings, vol. 8436, p. 84360C, SPIE Invited Paper, 2012.

[10] A. G. Marrugo, "https://github.com/agmarrugo/anisotropy-focus/",

[11] A. G. Marrugo, "http://www.goapi.upc.edu/usr/andre/sv-restoration/index.html", 